\title{
Don't post your videos and podcasts. Publish them. Cadmore Media: Streaming for scholarly and professional organizations
}

\author{
Violaine Iglesias ${ }^{\mathrm{a}, \mathrm{b}, *}$ \\ ${ }^{\mathrm{a}} \mathrm{CEO}$ and Founder, Cadmore Media, Bethesda, MD, USA \\ ${ }^{\mathrm{b}}$ Abingdon, $O X, U K$
}

\begin{abstract}
Why "publish" video and audio content? If you are a scholarly or professional organization, in all likelihood, your video and audio assets are authoritative sources of information. They should not just be posted online. They should be wellidentified, standardized, tagged, summarized, described, transcribed, segmented, translated, indexed, discovered, accessed, searched, skimmed, cited, clipped, embedded, shared, bookmarked, downloaded, saved, annotated. Just like journals, books, proceedings and, increasingly, datasets, scholarly and professional recordings should be published. Cadmore Media was founded to help.
\end{abstract}

Keywords: streaming media, video, podcasts, scholarly publishing, societies, professional associations, IGO, metadata standards, Cadmore Media

\section{Introduction: Quiz time!}

Since this is a write-up of a live presentation, let's start with an untraditional bang - a non-interactive, lengthy quiz:

Have you ever watched a video on the Internet? Have you ever not watched a video on the Internet because it was an hour long and you did not want to commit that much time to it? Have you ever stopped watching a video because you were not sure where it was going? Have you ever spent time rewinding and forwarding to try and find the one moment you might be interested in? Have you ever wondered how on earth one could find podcasts online?

Last, but not least: have you ever wished that the video you were watching was actually written text that you could read (or, more likely, skim)?

If you have answered yes to any of these questions, you are not alone. Please, read on.

E-mail: violaine@cadmore.media. 


\section{Streaming is on the rise, but what about scholarly communications?}

Cadmore Media was born out of a mission to serve the scholarly and professional world. Why then focus on less traditional formats? The answer is rather simple: in the consumer world, streaming is growing in popularity, for a variety of reasons. The Internet is becoming faster, mobile usage is exploding, and recording media is easier than ever before. Younger generations, of course, increasingly turn to YouTube for searches, rather than Google, which now makes the streaming platform the second most visited website in the world.

The higher education world has long embraced streaming, with academic libraries licensing video collections from a handful of specialized providers, and corporations have been turning to e-learning, but the scholarly and professional community has been slow to embrace audio and video. This is despite the many applications that can easily be listed for streaming. Not only is there a lot of untapped archival audiovisual content on hard drives all over the world, but more and more content is being produced every day: recorded events, research outputs, teaching and learning material, best practice videos, professional development courses, public outreach, podcasts. More often than not, this content lives on YouTube, making it impossible to distinguish authoritative content from amateur videos. Who wants their surgeon to "refresh their memory" on how to perform a procedure by watching a YouTube video? Well, at the moment, surgeons do use YouTube for professional purposes. It is not a stretch of the imagination to see a world where practitioners have access to curated, peer-reviewed videos published and vetted by society communities, who undoubtedly include the world's top experts.

In a scholarly world that is being shaken by new open access mandates, there is a need, and an opportunity, for non-profit organizations to diversify their sources of revenue and drive membership by proposing new types of content and benefits. Video and podcasts can be a natural part of that strategy.

\section{Unique challenges combined with a lack of infrastructure}

One reason why streaming has not been fully embraced is that it presents unique challenges.

While media content is a powerful way to communicate certain types of information, it is almost invariably hard to find. Once it has been found, the user experience is often unfriendly. Unlike with an article or a book, it is virtually impossible to know what a video or podcast contains without committing to watching or listening to it in its entirety. When it comes to entertainment, that is a perfectly desirable experience; for scholarly information, this can be a fastidious exercise for which most professionals do not have time. The human brain can read much faster than it can listen to someone speak to them. Most of us want the ability to skim a video in the same way that we would a long-form piece of written text.

Audiovisual content can also be difficult to understand without closed captions or a transcript: while the majority of the world's research is communicated in English, English is not the native language of the majority of researchers. Speakers can have strong accents (if you had heard this presentation, you would have heard mine), and listeners can have trouble picking up technical language in a tongue that is not theirs.

Crucially, multimedia content is often not accessible at all. Accessibility is still often an afterthought, even though the incentives to make content accessible - whether legal, commercial or moral - are becoming difficult to ignore. When it comes to video and audio content, not only is accessibility compliance the right thing to do, but it is also a sure way to boost searchability, indexability, discoverability, and general 
SEO. For more about the Far-Reaching Benefits of Multimedia Accessibility, see the author's article in the special January 2018 Accessibility edition of Learned Publishing (https://doi.org/10.1002/leap.1153).

Despite those challenges, there has not been a solution for publishers to handle video and audio content with the professionalism that has been awarded to digital books and journals for decades. Instead, there have only been mass-market solutions that are designed for large media organizations, but that are missing some key workflow pieces to fully integrate audiovisual material into the scholarly ecosystem, from peer-review to indexing and preservation. In particular, there has not been a good system to manage the collection and delivery of well-structured metadata.

All of these challenges, alongside other traditional obstacles to launching new programs such as the lack of budget, staffing - and sometimes an obvious ROI -, are making it difficult for scholarly and professional organizations to publish audio and video on a larger scale. This is not for lack of enthusiasm: virtually every organization we talk to has a desire to expand their reach in this area.

\section{Cadmore media, an end-to-end streaming platform for scholarly communications}

Years of market research and experience have led us to understand how to create a technical environment that addresses those challenges noted above. The most visible piece of that infrastructure is a "smart" media player that is platform-agnostic, accessible, multilingual, discoverable, responsive, and includes many features that enable faster navigation paths. Iterations of the player can be seen on our website's demo page: https://www.cadmore.media/playerdemo. Also, the Cadmore Media player is demonstrated with a video taken from a NASA science project at: https://app.cadmoremedia.com/VideoPlayer/Video/ BE6D1580-8FCD-46D2-9BE0-9A5D34E47B68.

In the backend, our platform provides media streaming and hosting, as well as - more uniquely workflow tools to help publishers enrich media assets with well-structured metadata, transcripts and segments, and to deliver that content and/or that newly-captured metadata to many different outlets, including publishing platforms, social media, discovery services, learning management systems and preservation tools. The platform also provides deep usage analytics, including industry-friendly ones such as COUNTER-compliant logs.

This vision is ambitious, and we do not intend to carry it out alone. Our technology is optimized for integration with third-party systems, including content repositories, workflow tools, publishing platforms and learning management systems. And our expertise is helping us lead industry-wide initiatives to improve the support for streaming content in scholarly and professional communications. For example, we are spearheading a NISO project to assess metadata standards for time-based media which will help publishers, vendors, and libraries to align their practices and streamline the discovery of streamed content (see: https://www.niso.org/press-releases/2019/05/niso-launches-new-project-developbest-practices-ensuring-findability-video).

\section{What makes us different from the competition?}

The reality is, there is no vendor with a similar offering. Of course, streaming is immediately associated with YouTube in most consumers' minds, but there are many reasons for a professional organization to be wary of using the social media network as its main media asset repository. For starters, any lawyer who assesses the platform's Terms of Use will balk at its unfavorable terms. And from a professional 


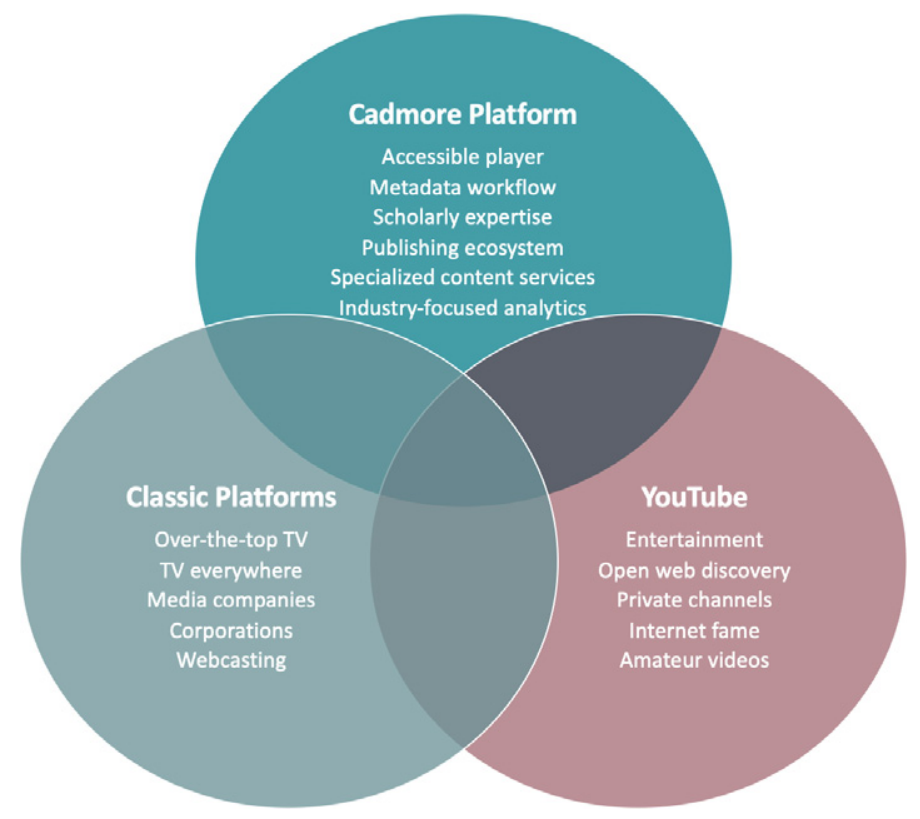

Fig. 1. Cadmore vs. our competitors.

user's perspective, it can be difficult to gauge the validity of any content that sits alongside a wide-range of amateur videos - remember that surgery example! And there are innumerable other arguments to not use YouTube as a content channel rather than as a content repository, from brand dilution to poor usage analytics outside of the main platform and lack of access control.

There are indeed generic streaming platforms that offer better terms of use, analytics, and security. However, the overlap between their services and Cadmore's is actually rather limited, and mainly cover the streaming technology itself, which has been commoditized over the past few years. Our metadata enrichment and delivery workflow, our understanding of scholarly and professional communications, and our network of specialized partners enable us to provide services that are much more targeted. DOI deposits, to just use one example, is not a service that any other streaming platform would offer.

\section{Cadmore's business model}

Since our participation at the 2019 NFAIS conference was in the context of a start-up challenge, we outlined our high-level business model for the audience. For our main platform, Cadmore offers a tiered subscription model that is based on a combination of media volume and usage. This is a model that is relatively standard and allows us to serve organizations of any size, smaller organizations that are just getting started to larger ones who already have significant volumes and/or usage. We focus on pricing transparency, and we do not impose any limit on embedding options for maximum flexibility.

In addition to the platform, we offer professional services for complex integrations, custom delivery targets and player customizations. And we have put partnership agreements in place that allow us to provide preferred pricing for content services such as transcription, metadata enrichment and translations. 


\section{About our Founders}

Cadmore Media was founded by scholarly communications professionals. We created the company in 2018 after researching the concept for a much longer time and discussing it with countless organizations. Our platform is now fully up and running.

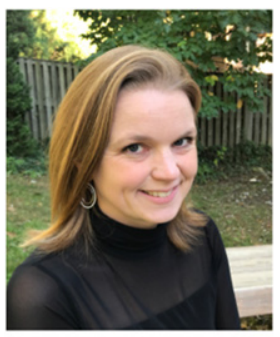

Violaine Iglesias CEO \& Co-founder

- Has built lots of video sites

- Loves plumbing metadata \& workflows

- Actually has a publishing degree

- Is conveniently American or French, depending on circumstances

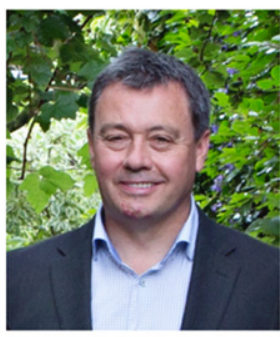

\section{Simon Inger}

Co-founder

- Launched the first online journal platform

- Knows a lot about societies (and cares about them)

- Wears lots of (metaphorical) hats

- Chops wood in his spare time

\section{About the Author}

Violaine Iglesias has experience that spans fifteen years in professional, scholarly and trade publishing. She started her career in book translation and trade publishing in Paris, France, before joining Random House in New York in 2007. She entered the scholarly communications world in 2011 at SAGE Publishing, where she oversaw the development of a wide-ranging suite of new digital reference products for academic libraries. In 2015, she joined technology company, GVPi, to advance its position as an independent provider of digital solutions for academic and professional publishers. In 2018, together with longtime industry consultant Simon Inger, she founded Cadmore Media with the aim to provide scholarly societies, professional associations, IGOs/NGOs and publishers with an expert environment to publish scholarly, professional and technical information through streaming video and audio. Phone: +1 (202) 641-6673; E-mail: violaine@cadmore.media; www.cadmore.media 